\title{
Technical Index Analysis and Structure Design Research of Modular Manipulators
}

\author{
$\mathrm{Yu} \mathrm{Bu}$ \\ School of Petroleum and Chemical Engineering, Dalian University of Technology, Dalian, 116000, \\ China
}

Keywords: Technical index, Structure design, Modular manipulators

\begin{abstract}
With the increasing demand of the human space mission, the manipulator plays an important role in the various tasks of space. In this paper, the technical indexes of modular manipulator, including joint torque index and joint accuracy index are given. According to the technical indexes of modular manipulator, we designed and developed the structure of modular manipulator to provide some references for the relative researchers.
\end{abstract}

\section{Introduction}

At present, the modular design method has been used in many industrial fields. The robot is a product of the combination of various new technologies, and the modular robot has become the focus of contemporary scholars. Mechanical arm modular joint is a mechanical structure. It is a drive in one of the mechatronic products in the design. We should not only consider the structure and size of the module, but also consider the good versatility and interchangeability in order to quickly assemble them into different configurations. The space detector with the manipulator not only has the ability to explore new things, but also has the ability to maintain the existing equipment. With the development of the docking technology of space robot and virtual intelligent control technology, the service will be further developed. It can be predicted that in the near future, the types of services that can be provided, and the services can be targeted and expanded. Manipulator technology is an important part of service technology, which has important research significance. As the only actuator of space service, the manipulator must have high reliability. The module and the system are equal to each other, and can realize the standardized interface of perfect connection. All modules have no effect on each other, but they can be connected to the integration function. The final finished product contains a large number of specific modules. We build the required products according to a series of different performance of different modules to realize the appropriate framework. Modular design emphasizes on the market forecast based on the analysis of product characteristics, the combination and exchange of different functional modules, the production of more flexible products in order to truly achieve the user's operating standards. The recycling strategy can increase the income of the enterprise and the adjustment and optimization of the module can stabilize the performance of the product.

\section{Technical Indexes of Modular Manipulators}

Joint Torque Index. We should make the action process of the modular manipulator in the task demonstration clear, analyze the worst working state in the process and carry on the mathematical calculation to determine the suitable load capacity index. The mechanical arm of the task execution is: first, the mechanical arm can be self-motion according to the acceleration and velocity; second, the arm in capturing the target platform will have an impact, especially in the target platform in a spin angular velocity; finally, when the manipulator to capture the target platform, the mechanical arm can drive the target platform moves at an angular velocity and angular acceleration. It is not difficult to find the above process. There are three possible limit loads in the working condition. The first is in the target platform before the mechanical arm with an angular acceleration movement; the second is to capture the target platform for the moment, the momentum of target platform manipulator was an instant absorption, which will impact the mechanical arm. Third, after the combination of success, 
even with a mechanical arm with the acceleration and velocity of the target platform for movement, the mechanical arm may appear extreme working condition, so the next will analyze these three conditions, the load demand of mechanical arm joint root. They adopt the structure of stepping motor and harmonic reducer, the drive and transmission unit of each joint are independent, which lays the foundation for the modularization of software. Stepper motor is characterized by high operating frequency, large output torque, suitable for high-speed operation. The stepping motor is embedded in the harmonic speed reducer, the structure is compact and the volume is small. The utility model is easy to assemble and disassemble, is easy to be aligned and clamped, and accurately transmits the movement and the power, and the two ends of the joint are connected with the connecting rod by a standardized internal and external thread.

Joint Accuracy Index. The common joint forms of industrial robots are moving joints and revolute joints. The most widely used industrial robot is a multi-joint robot, which is composed of a plurality of revolute joints and connecting rods, and simulates the functions of human shoulder joint, elbow joint and wrist joint. The joints are different from the shoulder, elbow and waist of humanoid robot. The moving joint is driven by a straight line, which includes the drive of the rectangular coordinate structure, the radial drive of the cylindrical coordinate structure and the vertical lifting drive, and the radial telescopic drive of the polar coordinate structure. Linear motion can be directly generated by the cylinder or hydraulic cylinder and piston. We can also use the gear rack, screw, nut and other transmission elements to convert the rotary motion into linear motion. A rotary joint is a joint mechanism which connects adjacent members, such as the arm and the base, the arm and the wrist, and the relative rotation or swing. Most of the motor can directly produce rotary motion, but often need a variety of gear, chain, belt drive or other gear reducer, in order to obtain a larger torque. Gear transmission is characterized by fast response, large torque, good rigidity, can be achieved to change the steering and composite transmission. The outputs of the two motors are respectively passed through the worm gear and the gear is transmitted through the middle gear. The utility model is composed of a wave generator, a flexible wheel and a rigid wheel. The utility model is characterized in that a flexible wheel is controlled by a wave generator to generate controllable elastic deformation. The utility model has the advantages of compact structure, large transmission ratio, high precision, high efficiency, coaxial line, simple structure and low rigidity. Currently, it is widely used in small and medium torque robot joints.

\section{Structure Design of Modular Manipulators}

Module of Base. With the shaft hole, the base can limit the direction of four degrees of freedom and check with pin. We can limit the degree of freedom of rotation of the sampling vessel, with the ball head spring pin and the annular groove is limited by the sampling of mobile container vertical degrees of freedom, such as sample containers fixed on the tracked robot platform. The ball head pin is pushed by the spring. When the sample container by force in vertical direction is certain and the ball head pin in the annular groove under the push, it will push the spring outward to automatically exit. The magnitude of this force can be achieved by selecting springs with different elastic forces. The base is the foundation of robot arm manipulator. In order to make the work smoothly, we need it to be able to bear larger overturning moment according to the technical requirements. Therefore, a reasonable structure is needed to ensure the movement performance of the whole machine. The collocation of motor planetary gear reducer with double reduction worm gear combination to promote rotator joint activities can guarantee the shoulder rotation at any angle joint no matter can achieve self-locking. The utility model contains a worm wheel and a worm control device, which belongs to a hollow double output shaft. The shoulder shaft and the base are connected by a series of bearings and oil seals, so as to minimize the loss of power transmission. The top of the shoulder shaft can be connected with other joint modules. As the task loads, the sampling tool and container need to be mounted on a tracked robot platform, but the wild environment is rough terrain in the process of fast moving sampling robot. The experiment will have different vibration amplitude and frequency, so the sample containers need reliable fixed on a mobile platform. After the completion of sampling, 
back to the rear of the operation, the sampling manipulator is to be able to easily remove the sampling vessel from the crawler robot platform to the staff. The sampling container is connected with the crawler robot platform through a fixed base.

Module of Grippers. The gripper module is indispensable part of end effector of the robot arm to capture the explosives. According to the characteristics of the captured object, all allowed distance and load grippers must meet the corresponding standards. At the same time, this paper points out that even the power is off, the object cannot be released. According to a number of domestic and foreign exploration results, the establishment of a more stable and reasonable structural treatment plan is implemented. The utility model is characterized that the hand clamping device is driven by a pot rod. When the motor drives the worm through the coupling, the rotating direction of the two turbines is opposite, namely, the opening and closing function of the gripper is realized. At the same time, the rubber is arranged at the jaw, which can further enhance the friction force between the object and the object to be processed. For solid ten extract, such as small animal and plant, it is similar to the tweezers tool to clip. It is different from the four kinds of tools and the tweezers sampling tools need a drive. The tweezers drive can be installed separately to drive the tweezers sampling tools. However, the power and control signal of the driver of the interface is more difficult to achieve, which will increase the cost. The gripper arm provides additional drive, when the gripper closed pushing inwards, so we can drive the tweezers grasp the target object through the lever mechanism. When the gripper is opened, the forceps open under the action of the spring.

Module of Joint. Joint module belongs to the support of the size of the arm rotation module. The adjustment of the end of the implementation of the location of the agency has an indispensable role. The operation of the arm of the size of the arm can enhance the scope and flexibility of the robot arm to capture the dangerous object in a timely and accurate. In dealing with the support module, we need to ensure that the weight of the item, the gripper, the rotary module will not exceed the allowable range of the module, and at the same time, we must ensure that the dynamic load can be accepted in the running phase. Since it belongs to the open chain, it is necessary to ensure the validity of the cross section of the boom, if we want to realize the accurate control and avoid the deformation during the pressure. The installation of a variety of screws will also cause the change in stiffness. Joint module quality should reach the standards. When the weight of the mechanical tissue is reduced, on the one hand, it can ensure the reasonable capture of the object, and the other one can reduce the moment of inertia of the manipulator itself and make the movement more harmonious. The joint module directly determines whether the robot arm can function properly and effectively or not. Through the coordination of different joints, in the process that the gripper is about to seize the object, it is necessary to accurately control the angle of the arm with the activity of the joint. Therefore, when the joint module activities, regardless of the angle at which the state can be stable, self-locking. A worm gear can control the motor speed to meet during the swing speed and the standards of output torque, which can achieve the functions accurate and timely self-locking.

Module of Link. The end of mechanical manipulator is one of the actuators. It has wide applicability to improve the general operation of the robot, according to the mechanical finger grip trajectory. The manipulator can be divided into single line, arc opening and closing type, parallel type, circular opening, closing opening and closing type. According to the power source of the manipulator, it can be divided into wire rope drive, motor drive and pneumatic drive. The manipulator should be able to capture the end of the sampling tool with the completion of the sampling tool for quick change, which requires the manipulator to open and close. In the field environment, air pressure or hydraulic power source is difficult to obtain. Therefore, the mechanical hand can only be driven by motor. The robot combines the characteristics of both the motor built-in manipulator, driving the screw rod to rotate through a gear, screw nut drive mobile, under the push rod to drive the parallelogram, open and closed. The motor is built to reduce the dimension of the vertical direction of the manipulator, which makes the whole structure more compact. The working space of biological sampling manipulator is composed of planar three link manipulator workspaces around the axis of rotation of the joint formation, and joint does not affect the end effector position, only rotation gripper, and therefore cannot be considered, so the analysis of biological sampling manipulator can focus on three link 
planar robot arms. The mechanical arm is composed of a space mechanism and kinematic pair, while kinematics modeling is a rigid rod connecting rod to be considered as the kinematics function. It is in the relative position of kinematic pair axis connecting the two ends. For the rotating joint, the offset of the connecting rod is fixed, and the angle of the connecting rod is variable.

\section{Conclusion}

Although the study of modular manipulator in our country started late, the researchers have made quite good achievements. In this paper, the base module, the joint module, the gripper module and the connecting rod module are designed in the process of the structure design of the modular manipulators. In the future, the link module will be designed further to trace the modular trend of the manipulator to make it more easily to be assembled.

\section{References}

[1] Lyu Jin, Zhang Jun, Wang Nan, K1nematics analysis of modular manipulators of mobile robot [J]. Hubei Journal of Industrial Science and Technology, 2013, 30(5): 333-337.

[2] Jiang Hongchao, Liu Shirong, Zhang Botao, Inverse kinematics analysis for 6 degree-of-freedom modular manipulator [J]. Journal of Zhejiang University (Engineering Science), 2010, 44(7): 1348-1354.

[3] Xu Wenfu, Du Xiaodong, Wang Chengjiang, Determination Method of Overall Technology Index for Space Manipulator [J]. Chinese Space Science and Technology, 2013(1): 53-60.

[4] Yan Jihong, Guo Xin, Liu Yubin, Zhao Jie, The design and kinematic analysis of a modular manipulator [J]. 2015, 47(1): 20-25. 